\title{
Fotocatálise do azul de metileno na presença de óxido de bismuto sob irradiação de luz UV e solar
}

\section{Methylene blue photocatalysis in the presence of bismuth oxide under UV and solar light irradiation}

\author{
Vanessa Rocha Liberatti ${ }^{1}$; Renata Afonso ${ }^{2}$; Adriana Campano Lucilha ${ }^{3}$; Paulo Rogério \\ Catarini da Silva ${ }^{4}$; Luiz Henrique Dall'Antonia ${ }^{5}$
}

\section{Resumo}

$\mathrm{O}$ óxido de bismuto $\left(\mathrm{Bi}_{2} \mathrm{O}_{3}\right)$, semicondutor do tipo- $n$, tem sido satisfatoriamente investigado para a remediação fotocatalítica de contaminantes orgânicos. $\mathrm{O} \mathrm{Bi}_{2} \mathrm{O}_{3}$ foi preparado por síntese de combustão em solução (SCS) utilizando como oxidante o nitrato de bismuto, em meio ácido, e a ureia como combustível. A influência da condição de síntese sobre as propriedades fotocatalíticas do óxido formado foi investigada por DRX. A partir dos difratogramas foi verificado que os materiais obtidos são predominantemente de cristais de $\mathrm{Bi}_{2} \mathrm{O}_{3}$, sendo possível identificar uma amostra com duas fases cristalinas, monoclínica $\left(\alpha-\mathrm{Bi}_{2} \mathrm{O}_{3}\right)$ e tetragonal ( $\beta$-Bi2O3), e a outra com apenas a fase monoclínica $\left(\alpha-\mathrm{Bi}_{2} \mathrm{O}_{3}\right)$. O óxido com duas fases apresentou maior atividade fotocatalítica para a descoloração do azul de metileno tanto sob irradiação UV $(60,59 \%)$ quanto sob luz solar $(61,64 \%)$, em $664 \mathrm{~nm}\left(\lambda_{\text {máx }}\right)$, seguindo lei cinética de pseudo-primeira ordem.

Palavras-chave: Fotocatálise. $\mathrm{Bi}_{2} \mathrm{O}_{3}$. Semicondutor.

\begin{abstract}
Bismuth oxide $\left(\mathrm{Bi}_{2} \mathrm{O}_{3}\right)$, an n-type semiconductor has been satisfactorily investigated for photocatalytic organic contaminant remediation. The $\mathrm{Bi}_{2} \mathrm{O}_{3}$ was prepared by solution combustion synthesis (SCS) using as the oxidizing bismuth nitrate in acidic medium and urea as fuel. The influence of the type of synthesis on the photocatalytic properties of the oxide formed was investigated by XRD. From the diffractograms was verified that the materials obtained are predominantly of $\mathrm{Bi}_{2} \mathrm{O}_{3}$ crystals, it is possible to identify a sample with two crystalline phases, monoclinic $\left(\alpha-\mathrm{Bi}_{2} \mathrm{O}_{3}\right)$ and tetragonal $\left(\beta-\mathrm{Bi}_{2} \mathrm{O}_{3}\right)$, and the other with only the monoclinic $\left(\alpha-\mathrm{Bi}_{2} \mathrm{O}_{3}\right)$. The two-phase oxide showed higher photocatalytic activity for discoloration of methylene blue under UV irradiation (60.59\%) and under sunlight (61.64\%) in 664 $\mathrm{nm}$, followed kinetic law of pseudo-first order.
\end{abstract}

Keywords: Photocatalysis. $\mathrm{Bi}_{2} \mathrm{O}_{3}$. Semiconductor.

Graduanda em Química, Universidade Estadual de Londrina.

2 Graduada em Química e aluna de doutorado em Química, Universidade Estadual de Londrina.

3 Graduada em Química e aluna de doutorado em Química, Universidade Estadual de Londrina.

4 Docente do Curso de Física, Universidade Estadual de Londrina.

5 Docente do Curso de Química, Universidade Estadual de Londrina; luizh@uel.com 


\section{Introdução}

A preocupação ambiental tem sido tema de muitos debates das organizações governamentais. Aliado a isso, a contaminação dos corpos de água e o consequente comprometimento destes têm levado a realização de estudos com o intuito de desenvolver tecnologias capazes de minimizar o impacto causado pela poluição no meio ambiente. Esta problemática ambiental associada ao efluente industrial é bastante conhecida e merece destaque devido a seu parque industrial gerar grandes volumes de efluentes contaminados (KUNZ et al., 2002) (ALMEIDA et al., 2004).

Diferentes corantes são utilizados para várias aplicações industriais e uma significativa proporção aparece na forma de efluentes. Estes efluentes são despejados nos corpos de água sem o devido tratamento, resultando em graves problemas estéticos (WONG; YU, 1999), além de alterar os ciclos biológicos afetando, principalmente, os processos fotossintéticos (SHEDBALKAR; DHANVE; JADHAV, 2008; NILSSON et al., 2006; MAZMANCI; ÜNYAYAR, 2005).

O corante azul de metileno, Colour Index (C.I.) 52015, é um composto aromático heterocíclico, com fórmula molecular: $\mathrm{C}_{16} \mathrm{H}_{18} \mathrm{ClN}_{3} \mathrm{~S} .3 \mathrm{H}_{2} \mathrm{O}$ e massa molar $373,86 \mathrm{~g} \mathrm{~mol}^{-1}$. É usado como corante bacteriológico e como indicador e sua absorção máxima em pH 5,6 é 664 nm.

A fotocatálise assume um papel importante na descontaminação de efluentes por ser uma técnica rápida e eficaz para a completa mineralização de contaminantes, através de espécies altamente oxidantes como radicais hidroxilas (HAMEED et al., 2009). O princípio da fotocatálise heterogênea envolve a ativação do semicondutor por luz solar ou artificial. A absorção de fótons com energia igual à energia de "bandgap" resulta na promoção de um elétron da banda de valência para a banda de condução com geração concomitante de uma lacuna $\left(h^{+}\right)$na banda de valência, a qual pode, subsequentemente, oxidar os contaminantes orgânicos (NOGUEIRA; JARDIM, 1998).
Semicondutores nanocristalinos fotoativos são empregados em sistemas como desinfecção de águas, sínteses orgânicas, células solares, sensores de gás, material para eletrodo positivo de baterias recarregáveis de lítio, entre outros (DALL' ANTONIA et al., 2010, KUDO; OMORI; KATO, 1999, SHANG et al., 2009). Embora semicondutores como $\mathrm{TiO}_{2}$ e $\mathrm{ZnO}$ tenham sido largamente investigados, estes não são fotocatalíticos quando excitados sob luz visível, um requisito essencial para utilização de energia solar. Entretanto, outros materiais capazes de fotoinduzir a separação de cargas, sob excitação na região visível do espectro eletromagnético, têm despertado grande interesse (LONG; CAI; KISCH, 2008). Um exemplo é o óxido de bismuto $\left(\mathrm{Bi}_{2} \mathrm{O}_{3}\right)$, material bastante utilizado devido as suas propriedades ópticas e elétricas, como energia de "bandgap", índice de refração, permissividade dielétrica, fotocondutividade e fotoluminescência (FAN et al., 2006). O óxido de bismuto é um semicondutor do tipo-n (LIN et al., 2007) utilizado na indústria de cerâmica, vidro e eletrônica, e na degradação de compostos orgânicos sob irradiação de luz visível. (LI; ZHANG; LIU, 2012).

$\mathrm{O}$ óxido de bismuto pode ser encontrado em cinco formas polimorfas conhecidas como $\alpha, \beta$, $\gamma, \delta$ e $\omega-\mathrm{Bi}_{2} \mathrm{O}_{3}$. Cada polimorfo possui estrutura cristalina e propriedades ópticas, elétricas e mecânicas diferentes (FAN et al., 2006). A forma $\alpha-\mathrm{Bi}_{2} \mathrm{O}_{3}$ apresenta eficiência fotocatalítica quando irradiada sob luz visível um requisito no que se refere à utilização de energia solar (LI; ZHANG; LIU, 2012; LI et al., 2011; EBERL; KISCH, 2008).

Muitas técnicas são empregadas para a síntese desses materiais fotoativos como, por exemplo, síntese em estado sólido (KUDO; OMORI; KATO, 1999), método sol-gel hidrólise (LIU; NAKAMURA; NAKATO, 2005), síntese por meio hidrotermal, síntese por precipitação em solução aquosa (ZHANG et al., 2007) e síntese de combustão em solução (JIANG et al., 2008), sendo que esta última tem despertado grande interesse para síntese 
de cerâmica, ligas, intermetálicos, nanomateriais, catálises e compósitos.

A síntese de combustão em solução (SCS) tem sido muito utilizada por, além de ser um processo simples, rápido e versátil, liberar quantidade de energia suficiente para a produção de cristais finos, puros, homogêneos e em escala nanométrica. (JIANG et al., 2008, TIMMAJI et al., 2011, JIANG et al., 2009). Assim, este trabalho tem como objetivo investigar a influência da condição de síntese sobre as propriedades fotocatalíticas de $\mathrm{Bi}_{2} \mathrm{O}_{3}$ e comparar a utilização da luz UV e solar na descoloração do azul de metileno.

\section{Parte Experimental}

\section{Materiais}

O corante azul de metileno $\left(\mathrm{C}_{16} \mathrm{H}_{18} \mathrm{ClN}_{3} \mathrm{~S} .3 \mathrm{H}_{2} \mathrm{O}\right)$, C. I. 52015 foi utilizado, sem purificação prévia. Todos os reagentes utilizados para a síntese do óxido de bismuto e para a fotocatálise heterogênea apresentaram grau analítico. Todas as soluções foram preparadas com água ultrapura (Millipore Plus).

Os reagentes utilizados para a síntese de combustão em solução do óxido de bismuto foram nitrato de bismuto III pentahidratado $\left(\mathrm{Bi}\left(\mathrm{NO}_{3}\right)_{3} .5 \mathrm{H}_{2} \mathrm{O}\right.$, Vetec, $\left.98 \%\right)$, ureia $\left(\mathrm{NH}_{2} \mathrm{CONH}_{2}\right.$, Cinética Química, 99\%) e ácido nítrico $\left(\mathrm{HNO}_{3}\right.$, Biotec, 65\%).

\section{Síntese de combustão em solução}

O óxido de bismuto foi obtido por síntese de combustão em solução (SCS) a partir de $50 \mathrm{~mL}$ de solução $25 \mathrm{mM}$ de nitrato de bismuto em meio ácido. A solução foi mantida sob agitação magnética, para completa dissolução do nitrato de bismuto em $\mathrm{HNO}_{3}$. Em seguida foi adicionado 0,5 $\mathrm{g}$ de ureia, utilizada como combustível na síntese.

A primeira mistura (1) foi deixada em estufa (Brasdonto modelo 5) por $20 \mathrm{~h}$ a $80^{\circ} \mathrm{C}$ até a formação de um gel e em seguida calcinada a $500 \pm 2{ }^{\circ} \mathrm{C}$ por $1 \mathrm{~h}$ em mufla (EDG Equipamentos EDGCON 1P), formando um pó amarelo. A segunda mistura (2) foi deixada sob refrigeração a $4{ }^{\circ} \mathrm{C}$ por 15 dias e depois seguido o mesmo procedimento da mistura 1 .

\section{Caracterização do semicondutor $\mathrm{Bi}_{2} \mathrm{O}_{3}$ por difração de raios $X$}

A estrutura cristalina das duas amostras foi investigada por difração de raios X (DRX). Os difratogramas foram obtidos pelo método de pó, em um difratômetro da PANalytical modelo X'Pert PRO MPD, utilizando radiação $\mathrm{CuK \alpha}(1,5418$ $\AA$ ), com tensão de $40 \mathrm{kV}$ e corrente de $30 \mathrm{~mA}$. As amostras foram analisadas e autosuportadas em porta-amostras de aço inox. Após as medidas foi aplicado o Método Rietveld de refinamento da estrutura cristalina nos difratogramas, para se determinar os parâmetros de rede e percentagens entre fases observadas.

\section{Fotocatálise heterogênea do azul de metileno}

$50 \mathrm{~mL}$ da suspensão formada por $0,02 \mathrm{mmol} \mathrm{L}^{-1}$ de azul de metileno e $2,0 \mathrm{~g} \mathrm{~L}^{-1}$ do fotocatalisador $\left(\mathrm{Bi}_{2} \mathrm{O}_{3}\right)$ foi deixada no banho ultrassônico por 30 min a $25{ }^{\circ} \mathrm{C}$ e depois exposta à luz por $180 \mathrm{~min}$. As irradiações das amostras por luz UV foram realizadas em béquer, no interior de uma caixa de madeira $(36 \times 39 \times 67 \mathrm{~cm})$ com ventilação, revestida internamente com papel alumínio, usando-se lâmpada de vapor de $\mathrm{Hg}$ sem bulbo de $250 \mathrm{~W}$ $\left(0,160 \mathrm{~mW} \mathrm{~cm}^{-2}\right)$, posicionada a $30 \mathrm{~cm}$ da suspensão mantida sob agitação magnética (Tecnal modelo TE 085) em $27 \pm 2{ }^{\circ} \mathrm{C}$. Já a irradiação sob luz solar foi realizada em frasco scott no período do dia de maior intensidade solar, ou seja, das $11 \mathrm{~h} 20 \mathrm{~min}$ às 14h20min, em 03/07/2012 com irradiação solar média de $43,8 \mathrm{~mW} \mathrm{~cm}{ }^{-2}$, em $27 \pm 2{ }^{\circ} \mathrm{C}$. As alíquotas foram retiradas em intervalo de $30 \mathrm{~min}$, centrifugadas por $3 \mathrm{~min}$ (Centribio modelo 80-2b) e imediatamente analisadas por espectrofotometria no visível de 350 a 800 nm (Ocean Optics USB4000) em $664 \mathrm{~nm}$. 


\section{Resultados e discussão}

Na Figura 1 são mostrados os difratogramas de raios $\mathrm{X}$ das amostras estudadas onde se observa que o óxido de bismuto preparado a partir da solução 1 possui duas fases cristalográficas: uma monoclínica (PDF $n^{\circ}$ 01-076-1730) e uma tetragonal (PDF $n^{\circ}$ 01-076-0147) (Figura 1A), já o óxido de bismuto preparado a partir da solução 2 possui apenas a fase monoclínica, sendo que todos os picos observados se referem a fase $\left(\alpha-\mathrm{Bi}_{2} \mathrm{O}_{3}\right)\left(\mathrm{PDF} \mathrm{n}^{\circ}\right.$ 01-071-2274) (Figura 1B).

Figura 1 - Difratogramas de raios $\mathrm{X}$ do óxido de bismuto obtido por síntese de combustão a partir da (A) solução 1 e (B) solução 2 , em que $\left(^{*}\right)$ indica a fase monoclínica $\left(\alpha-\mathrm{Bi}_{2} \mathrm{O}_{3}\right)$ e $\left({ }^{\circ}\right)$ indica a fase tetragonal $\left(\beta-\mathrm{Bi}_{2} \mathrm{O}_{3}\right)$.

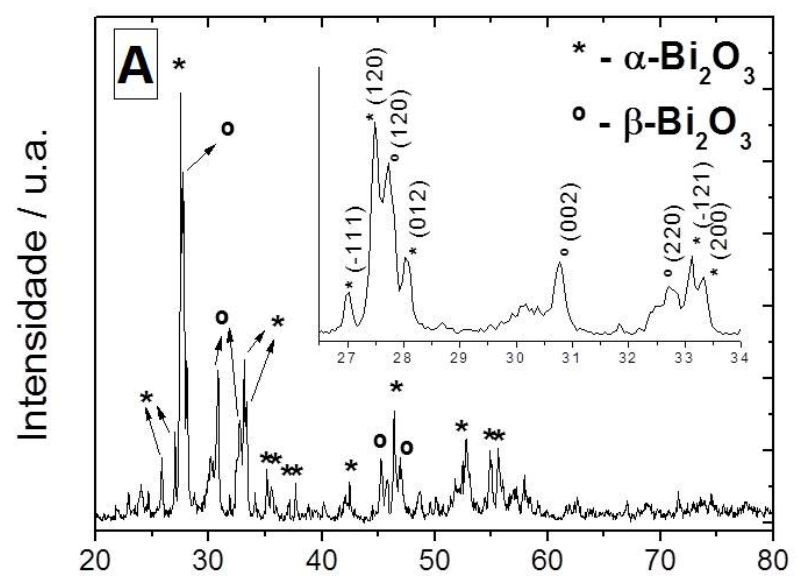

Fonte: Autores

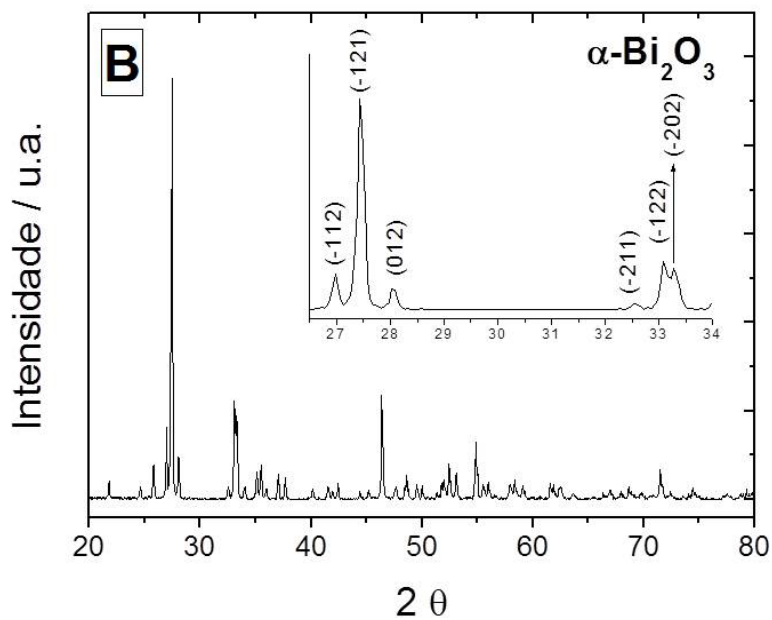

A razão entre a intensidade dos picos e os ruídos observados nos difratogramas mostram que o óxido obtido pela solução 2 possui cristais maiores e bem formados, devido aos picos estreitos e intensos. Para se determinar os parâmetros de rede e as proporções entre as fases observadas na Figura 1, foi utilizado o Método Rietveld de refinamento da estrutura cristalina, e os resultados são mostrados nas tabelas 1, 2 e 3. Os parâmetros de rede dos padrões e do óxido de bismuto obtido por SCS a partir das soluções 1 e 2 são apresentados nas Tabelas 2 e 3, respectivamente.

Tabela 1 - Porcentagem de fase e grupo espacial do óxido de bismuto obtido por SCS a partir da solução 1.

\begin{tabular}{cccc}
\hline Fase & $\begin{array}{c}\text { Sistema } \\
\text { cristalino }\end{array}$ & $\begin{array}{c}\text { Porcentagem } \\
\text { da fase }\end{array}$ & $\begin{array}{c}\text { Grupo } \\
\text { espacial }\end{array}$ \\
\hline$\alpha-\mathrm{Bi}_{2} \mathrm{O}_{3}$ & Monoclínica & $34,7 \%$ & $\mathrm{P} 2{ }_{1} / \mathrm{c}$ \\
$\beta-\mathrm{Bi}_{2} \mathrm{O}_{3}$ & Tetragonal & $65,3 \%$ & $\mathrm{P}-42{ }_{1} \mathrm{c}$ \\
\hline
\end{tabular}

Fonte: Autores

Tabela 2 - Parâmetros de rede dos padrões e do óxido de bismuto obtido por SCS a partir da solução 1.

\begin{tabular}{ccccc}
\hline $\begin{array}{c}\text { Parâmetros } \\
\text { de cela }\end{array}$ & $\alpha-\mathrm{Bi}_{2} \mathrm{O}_{3}$ & $\begin{array}{c}\mathrm{Padrão} \\
\alpha-\mathrm{Bi}_{2} \mathrm{O}_{3}\end{array}$ & $\beta-\mathrm{Bi}_{2} \mathrm{O}_{3}$ & $\begin{array}{c}\mathrm{Padrão} \\
\beta-\mathrm{Bi}_{2} \mathrm{O}_{3}\end{array}$ \\
\hline $\mathrm{a}$ & $5,847 \AA$ & $5,840 \AA$ & $7,748 \AA$ & $7,738 \AA$ \\
$\mathrm{b}$ & $8,163 \AA$ & $8,160 \AA$ & $7,747 \AA$ & $7,738 \AA$ \\
$\mathrm{c}$ & $7,501 \AA$ & $7,490 \AA$ & $5,824 \AA$ & $5,731 \AA$ \\
$\alpha$ & $90^{\circ}$ & $90^{\circ}$ & $90^{\circ}$ & $90^{\circ}$ \\
$\beta$ & $67,09^{\circ}$ & $112,93^{\circ}$ & $90^{\circ}$ & $90^{\circ}$ \\
$\gamma$ & $90^{\circ}$ & $90^{\circ}$ & $90^{\circ}$ & $90^{\circ}$ \\
$\begin{array}{c}\text { Volume } / \\
\mathrm{pm}^{3}\end{array}$ & 329,8 & & 349,6 & \\
\hline
\end{tabular}

Fonte: Autores

Fonte: Autores 
Tabela 3 - Parâmetros de rede do padrão e do óxido de bismuto obtido por SCS a partir da solução 2 .

\begin{tabular}{ccc}
\hline $\begin{array}{c}\text { Parâmetros } \\
\text { de cela }\end{array}$ & $\alpha-\mathrm{Bi}_{2} \mathrm{O}_{3}$ & $\begin{array}{c}\mathrm{Padrão} \\
\alpha-\mathrm{Bi}_{2} \mathrm{O}_{3}\end{array}$ \\
\hline $\mathrm{a}$ & $5,847 \AA$ & $5,849 \AA$ \\
$\mathrm{b}$ & $8,165 \AA$ & $8,166 \AA$ \\
$\mathrm{c}$ & $7,509 \AA$ & $7,510 \AA$ \\
$\alpha$ & $90^{\circ}$ & $90^{\circ}$ \\
$\beta$ & $112,98^{\circ}$ & $113,00^{\circ}$ \\
$\gamma$ & $90^{\circ}$ & $90^{\circ}$ \\
Volume $/ \mathrm{pm}^{3}$ & $330,0 \times 10^{6}$ & \\
\hline
\end{tabular}

Fonte: Autores

Como pode se observar na Tabela 1, o óxido obtido a partir da solução 1 possui uma maior quantidade de material na fase tetragonal, sendo $\mathrm{P}-42{ }_{1} \mathrm{c}$ o seu grupo espacial. No óxido obtido através da solução 2 , embora formado somente pela fase $\alpha-\mathrm{Bi}_{2} \mathrm{O}_{3}$ (monoclínica), foi observado que o seu grupo espacial é o mesmo da fase monoclínica do óxido obtido a partir da solução $1, \mathrm{P} 2_{1} / \mathrm{c}$.

Os difratogramas calculados a partir do método Rietveld se ajustaram bem às medidas, como pode ser observado na Figura 2. Fato que se deve a grande quantidade de material cristalino e principalmente ao baixo valor no teste do $\chi 2$ (teste utilizado para verificação da correspondência entre o valor medido e calculado), sendo de 2,913 para o $\mathrm{Bi}_{2} \mathrm{O}_{3}$ obtido a partir da solução 1 e de 1,761 para o $\mathrm{Bi}_{2} \mathrm{O}_{3}$ obtido a partir da solução 2 .
Figura 2 - Difratogramas de raios $\mathrm{X}$ medido, calculado, a diferença entre eles e o background para o óxido obtido a partir da (A) solução 1 e (B) solução 2 .

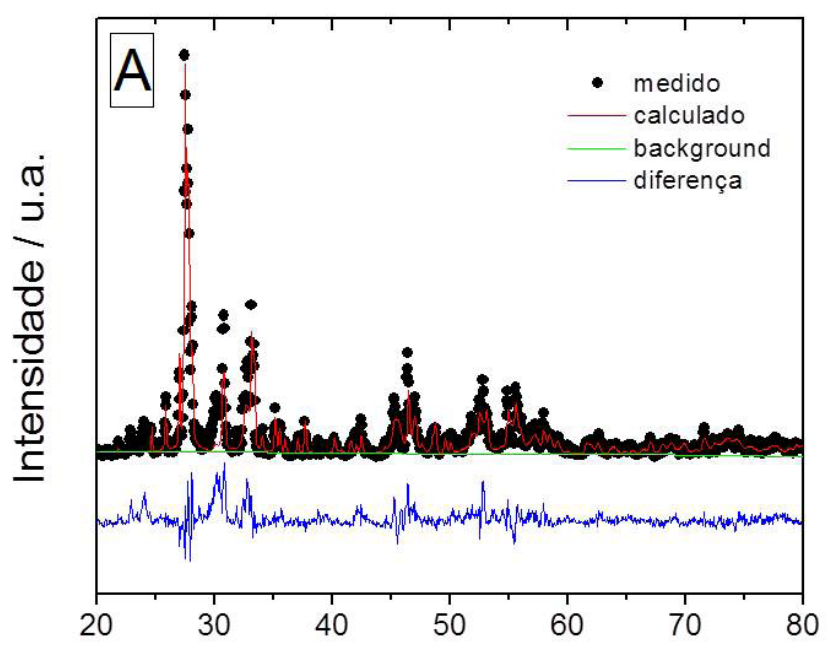

Fonte: Autores

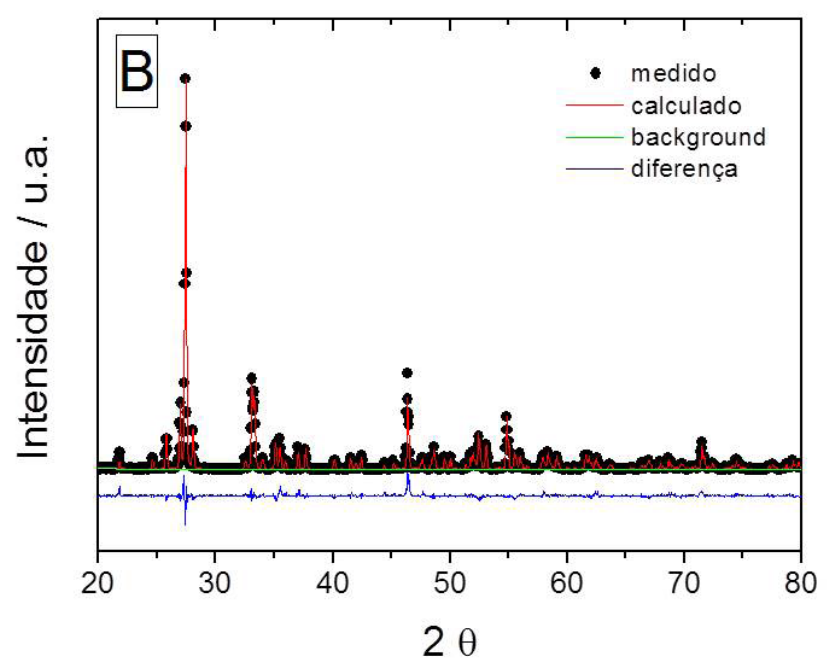

Fonte: Autores

O estudo da fotocatálise foi observado pela descoloração da solução do corante azul de metileno, através da diminuição da absorvância no espectro visível, no comprimento de onda de $664 \mathrm{~nm}$.

Os espectros das soluções de descoloração do azul de metileno mostraram que a absorvância em $664 \mathrm{~nm}$ diminui com o tempo, tanto com a utilização da luz solar como com a luz ultravioleta. 
A reação de descoloração do corante azul de metileno seguiu uma cinética de pseudo-primeira ordem, dada pela equação (1):

$$
\ln \left(A b s_{t}\right)-\ln \left(A b s_{0}\right)=-k t
$$

em que $\mathrm{Abs}_{\mathrm{t}}$ é a absorvância da solução no tempo t, $\mathrm{Abs}_{0}$ é a absorvância da solução no tempo zero, $\mathrm{k}$ é a constante de velocidade (tempo ${ }^{-1}$ ) e t é o tempo.

Os coeficientes de correlação linear $\left(\mathrm{R}_{2}\right)$ e as constantes de velocidade $\left(k_{o b s}\right)$ calculadas para a descoloração do azul de metileno pelo óxido de bismuto obtido pelas soluções 1 e 2 são mostrados na Tabela 4.

Tabela 4 - Parâmetros cinéticos para a descoloração do azul de metileno em luz UV e solar obtidos a partir do óxido de bismuto com duas e uma fase.

\begin{tabular}{lccc}
\hline Fotocatalisador & $\mathrm{Luz}$ & $k_{o b s} / 10^{-3} \mathrm{~min}^{-1}$ & $\mathrm{R}^{2}$ \\
\hline & $\mathrm{UV}$ & 6,11 & 0,948 \\
$\mathrm{Bi}_{2} \mathrm{O}_{3}$ (duas fases) & Solar & 6,56 & 0,987 \\
& $\mathrm{UV}$ & 1,98 & 0,866 \\
$\mathrm{Bi}_{2} \mathrm{O}_{3}$ (uma fase) & Solar & 3,93 & 0,974 \\
\hline
\end{tabular}

Fonte: Autores

Os dados mostram que a constante de velocidade da reação de descoloração utilizando o óxido com duas fases foi maior com luz solar do que com luz ultravioleta. A percentagem de redução da cor da solução de azul de metileno foi de $61,64 \%$ com luz solar e 60,59 \% com luz ultravioleta. Já a constante de velocidade da reação de descoloração com o óxido com apenas uma fase também foi maior com luz solar do que com a luz ultravioleta. A percentagem de descoloração do azul de metileno foi de $37,73 \%$ com luz solar e de $26,74 \%$ com a luz ultravioleta.
Essa pequena diferença da percentagem de descoloração da solução de azul de metileno usando as duas formas de radiação pode ser explicada pelo fato de tanto a forma $\alpha-\mathrm{Bi}_{2} \mathrm{O}_{3}$ quanto a forma $\beta-\mathrm{Bi}_{2} \mathrm{O}_{3}$ absorverem radiação na região da luz visível, uma vez que os valores de energia de bandgap encontrado na literatura são $2,8 \mathrm{eV}$ para a $\alpha-\mathrm{Bi}_{2} \mathrm{O}_{3}$ e $2,3 \mathrm{eV}$ para a $\beta-\mathrm{Bi}_{2} \mathrm{O}_{3}$ (EBERL; KISCH, 2008).

\section{Conclusões}

O óxido que apresentou apenas a fase alfa teve diferença significativa entre a descoloração feita a partir da luz solar em comparação com a luz ultravioleta. Porém, se comparado com o óxido de duas fases, percebeu-se que a descoloração foi ainda maior, tanto na luz ultravioleta quanto na luz solar. Portanto, a mistura do $\alpha-\mathrm{Bi}_{2} \mathrm{O}_{3}$ com a fase $\beta-\mathrm{Bi}_{2} \mathrm{O}_{3}$ é vantajoso na descoloração do corante utilizado.

Os resultados obtidos pela luz UV e solar são muito semelhantes no óxido que contém a mistura das fases alfa e beta. A luz solar teve minimamente melhores resultados de descoloração do corante azul de metileno que a luz UV (com diferença de aproximadamente $1 \%$ ), portanto o uso da luz solar é mais viável por estar disponível sem nenhum custo.

\section{Agradecimentos}

Nossos agradecimentos à Universidade Estadual de Londrina (UEL), à Fundação Araucária e ao CNPq pelo apoio financeiro. À Fundação Araucária e CAPES, pela bolsa concedida aos alunos.

\section{Referências}

ALMEIDA, E.; ASSALIN, M. R.; ROSA, M. A.; DURAN, N. Tratamento de efluentes industriais por processos oxidativos na presença de ozônio. Química Nova, v. 27, n. 5, p. 818-824, jul. 2004. 
DALL' ANTONIA, L. H.; TACCONI, N. R. de; CHANMANEE, W.; TIMMAJI, H.; MYUNG, N.; RAJESHWAR, K. Electrosynthesis of bismuth vanadate photoelectrodes. Electroc. and Solid-State Letters, v. 13, p. 29-32, 2010.

EBERL, J.; KISCH, H.; Visible light photooxidations in the presence of $\alpha-\mathrm{Bi}_{2} \mathrm{O}_{3}$. Photochemical \& Photobiological Sciences, v. 7, n. 11, p. 14001406, 2008.

FAN, H. T.; PAN, S. S.; TENG, X. M.; YE, C.; LI, G. H.; ZHANG, L. D. $\alpha-\mathrm{Bi}_{2} \mathrm{O}_{3}$ thin films prepared by reactive sputtering: Fabrication and characterization. Thin Solid Films, v. 513, n. 1, p. 142-147, 2006.

HAMEED, A.;GOMBAC, V.; MONTINI, T.; GRAZIANI, M.; FORNASIERO, P. Synthesis, characterization and photocatalytic activity of NiO$\mathrm{Bi}_{2} \mathrm{O}_{3}$ Nanocomposites. Chemical Physics Letters, v. 472, p. 212-216, 2009.

JIANG, H.; ENDO, H.; NATORI, H.; NAGAI, M.; KOBAYASHI, K.; Fabrication and photoactivities of spherical-shaped $\mathrm{BiVO}_{4}$ photocatalysts through solution combustion syntesis method. J. Europen Ceramic Society, v. 28, n. 15, p. 2955 - 2962, 2008.

JIANG, H.; ENDO, H.; NATORI, H.; NAGAI, M.;. KOBAYASHI, K. Fabrication and efficient photocatalytic degradation of methylene blue over $\mathrm{CuO} / \mathrm{BiVO}_{4}$ composite under visible-light irradiation. Materials Research Bulletin, v. 44, n. 3, p. 700-706, 2009.

KUDO, A.; OMORI, K.; KATO, H. A novel aqueous process for preparation of crystal form-controlled and highly crystalline $\mathrm{BiVO}_{4}$ powder from layered vanadates at room temperature and its photocatalytic and photophysical properties. J. Europen Ceramic Society, v. 121, p. 11459-11467, 1999.

KUNZ, A.; PERALTA-ZAMORA, P.; MORAES, S. G.; DURAN, N. Novas tendências no tratamento de efluentes têxteis. Química Nova, v. 25, n. 1, p. 78-82, 2002.
LI, C.; ZHANG, J.; LIU, K.; A new method of enhancing photoelectrochemical characteristics of $\mathrm{Bi} / \mathrm{Bi}_{2} \mathrm{O}_{3}$ electrode for hydrogen generation via water splitting. International Journal of Electrochemical Science, v. 7 , p. 5028-5034, 2012.

LI, E-J.; XIA, K.; YIN, S-F.; DAI, W-L.; LUO, S-L.; AU, C-T. Preparation, characterization and photocatalytic activity of $\mathrm{Bi}_{2} \mathrm{O}_{3}-\mathrm{MgO}$ composites. Materials Chemistry and Physics, v. 125, p. 236-241, 2011.

LIN, X.; XING, J.; WANG, W.; SHAN, Z.; XU, F.; HUANG, F. Photocatalytic activities of heterojunction semiconductors $\mathrm{BI}_{2} \mathrm{O}_{3} / \mathrm{BaTiO}_{3}$ : a strategy for the design of efficient combined photocatalysts. Journal of Physical Chemistry C., v. 111, p. 18288 - 18293, 2007.

LIU, H.; NAKAMURA, R.; NAKATO, Y. Promoted photo-oxidation reactivity of particulate $\mathrm{BiVO}_{4}$ photocatalyst prepared by a photoassisted sol-gel method. Journal of the Electrochemical Society, v. 152, p. 856-861, 2005.

LONG, M.; CAI, W.; KISH, H. Visible light induced photoelectrochemical properties of $\mathrm{n}-\mathrm{BiVO}_{4}$ and $\mathrm{n}-\mathrm{BiVO}_{4} / \mathrm{p}-\mathrm{Co}_{3} \mathrm{O}_{4}$. Journal of Physical Chemistry C., v. 112, p. 548-554, 2008.

MAZMANCI, M. A.; ÜNYAYAR, A. Decolourisation of reactive black 5 by funalia trogii immobilized on luffa cylindrical sponge. Process Biochemistry, v. 40, p. 337342, 2005.

NILSSON, I.; MÖLLER, A.; MATTIASSON, B.; RUBINDAMAYUGI, M. S. T.; WELANDER, U. Decolorization of synthetic and real textile wastewater by the use of white-rot fungi. Enzyme and Microbial Technology, v. 38, p. 94-100, 2006.

NOGUEIRA, R. F. P.; JARDIM, W. F.; A fotocatálise heterogênea e sua aplicação ambiental. Quimica Nova, v. 21, n. 1, p. 69-72, 1998.

SHANG, M.; WANG, W.; SUNG, S.; REN, J.; ZHOU, L.; ZHANG, L. Efficient visible light-induced photocatalytic degradation of contaminant by spindlelike PANI/BiVO, . Journal of Physical Chemistry C, v. 113, p. 20228-20333, 2009. 
SHEDBALKAR, U.; DHANVE, R.; JADHAV, J. Biodegradation of triphenylmethane dye cotton blue by penicillium ochrochloron MTCC 517. Journal of Hazardous Materials, v. 157, p. 472-479, 2008.

TIMMAJI, H. K.; CHANMANEE, W.; TACCONI N. R. de; RAJESHWAR K. Solution combustion synthesis of $\mathrm{BiVO}_{4}$ nanoparticles: effect of combustion precursors on the photocatalytic activity. Science \& Technology Network, Inc. v. 14, n. 1, p. 93-105, 2011.

WONG, Y.; YU, J. Laccase-catalyzed decolorization of synthetic dyes. Water Research, v. 33, p. 35123520, 1999.

ZHANG, X.; AI, Z.; JIA, F.; ZHANG, L.; FAN. $\mathrm{X}$.; ZOU, Z. Selective synthesis and visible-light photocatalytic activities of $\mathrm{BiVO}_{4}$ with different crystalline phases. Mat. Chem. and Physics, v. 103, p. 162-167, 2007.

Recebido em 4 Fevereiro 2013-Received on February 4, 2013. Aceito em 10 Fevereiro, 2014 - Accepted on February 10, 2014. 